Check for updates

Cite this: RSC Adv., 2020, 10, 17854

\title{
Effect of new carbonyl cyanide aromatic hydrazones on biofilm inhibition against methicillin resistant Staphylococcus aureus $\dagger$
}

\author{
Xueer Lu, ${ }^{\text {ab }}$ Ziwen Zhang, ${ }^{c}$ Yingying $\mathrm{Xu}^{\mathrm{c}}{ }^{\mathrm{c}}$ Jun Lu, ${ }^{\mathrm{a}}$ Wenjian Tang (D) ${ }^{\mathrm{c}}$ \\ and Jing Zhang (D) *ab
}

Carbonyl cyanide $m$-chlorophenylhydrazone (CCCP), as a protonophore, in combination with antibiotics exhibited potentiating antibacterial activity. To improve CCCP's potency and toxicity, a series of aromatic hydrazones were synthesized and their antimicrobial activity was evaluated; amongst them, compounds $2 \mathrm{e}$ and $2 \mathrm{j}$ with a strong para-electron-withdrawing substituent $\left(-\mathrm{NO}_{2}\right.$ and $\left.-\mathrm{CF}_{3}\right)$ at the phenyl ring had the lowest MICs against both S. aureus and methicillin resistant Staphylococcus aureus (1.56 and 1.56 $\mu \mathrm{M}$, respectively). Some compounds in combination with antibiotics exhibited potentiate Gram-positive antibacterial activity; compound $2 \mathrm{e}$ was found to display unaided or synergistic efficacy against MRSA. In particular, when compound $2 \mathrm{e}$ is combined with ofloxacin, it has a good synergistic effect against MRSA. Moreover, electron microscopy revealed that compound $2 \mathrm{e}$ inhibits biofilm formation and effectively eradicates preformed biofilm. MTT assay showed that compound $2 \mathrm{e}$ displays as low toxicity as CCCP. Overall, our data showed that the aromatic hydrazone is a promising scaffold for anti-staphylococcal drug development.

Received 7th April 2020

Accepted 29th April 2020

DOI: $10.1039 / \mathrm{dOra03124k}$

rsc.li/rsc-advances

uncoupling of the proton gradient, acts essentially as an ionophore and reduces the ability of ATP synthase to function optimally. CCCP causes the gradual destruction of living cells and death of the organism by affecting the respiration and respiration-dependent phosphorylation. ${ }^{8,9}$ Antibiotic accumulation in Gram-negative bacteria is one of the major causes of AMR. CCCP was widely used to study cellular accumulation in Gram-negative bacteria for many small molecules ${ }^{10-12}$ due to its ability to collapse the proton motive force. ${ }^{13}$ As AMR spreads, a promising approach is to restore the effectiveness of existing drugs via co-administration with adjuvants that inhibit the growth of drug-sensitive pathogens. ${ }^{4,14,15}$ CCCP in combination with small molecules showed synergistic effect against most of the MDR pathogenic bacterial strains. ${ }^{16-20} \mathrm{CCCP}$ in combination with antibiotics could potentiate antibacterial activity.

Since CCCP acts as a protonophore which disperses the membrane proton motive force by modifying the transmembrane electrochemical potential, it simultaneously causes toxicity to the cell of the host. ${ }^{21,22}$ Moreover, the concentration of synergistic antibacterial effect of CCCP is so high (at least $50 \mu \mathrm{M}$ ) that the effective dose may disrupt mitochondrial function to lead to toxicity. ${ }^{23-25}$ Therefore, in this work, a series of aromatic hydrazones were synthesized and evaluated for their antibacterial activity to try and improve antibacterial potency and reduce toxicity. The preliminary screening showed that aromatic hydrazones exhibited potential Gram-positive antibacterial activities. New compounds alone or in combination with antibiotics exhibited potentiate Gram-positive antibacterial activities. 


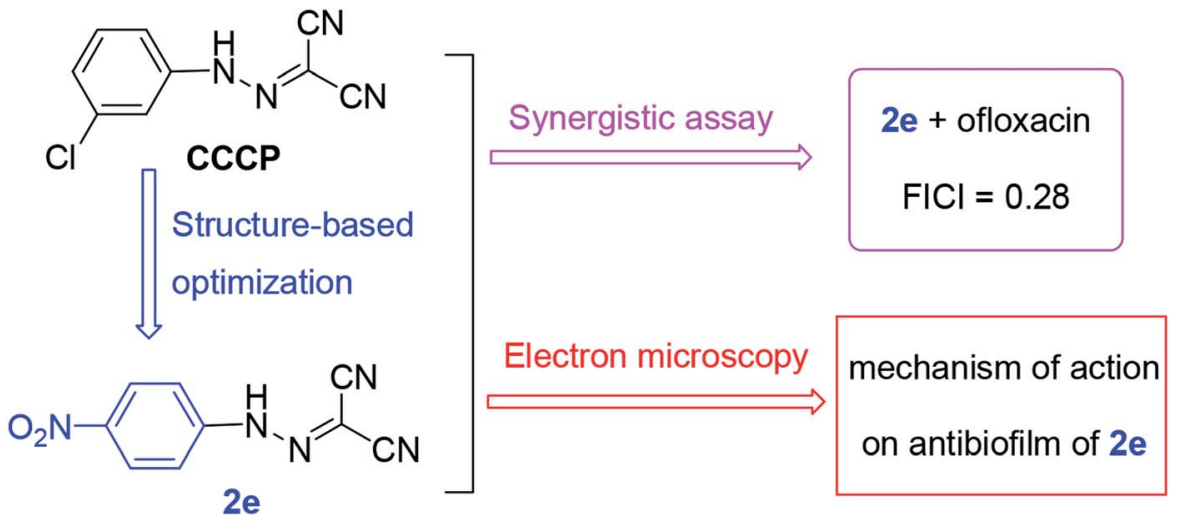

Fig. 1 The general strategy in this work.

Therefore, the aromatic residue is a promising scaffold for further antibacterial modifications. Further, a plausible antibacterial mechanism was proposed and investigated via scanning electron microscopy (SEM) and transmission electron microscopy (TEM) (Fig. 1).

\section{Results and discussion}

\subsection{Chemistry}

The synthetic route to compounds $\mathbf{2 a - 2 q}$ is illustrated in Scheme 1. The nitrosation of the aromatic amines (1) with nitrous acid (in situ from sodium nitrite and concentrated hydrochloric acid) led to aromatic diazonium salts, which can be used to next reaction without purification. The diazonium salt as the key intermediate underwent a condensation reaction with methylene of malononitrile to yield the title compound 2 .

\subsection{Antibacterial activity of compounds $2 a-2 q$}

In order to determine the antimicrobial potential of aromatic hydrazones, they were evaluated in either Mueller-Hinton (MH) broth or Sabouraud Dextrose Agar (SDA) using a micro-broth dilution method against a panel of bacteria and fungi, including two Gram-positive bacteria: Staphylococcus aureus ATCC 25923 (SA) and Methicillin Resistant Staphylococcus

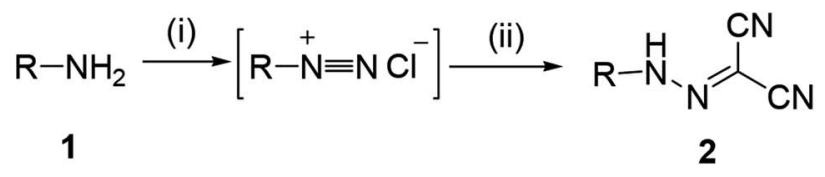

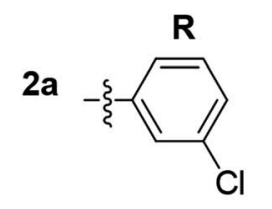

$2 \mathbf{b}$<smiles>Cc1ccnc(Cl)c1</smiles>

2c<smiles>COc1cc(C)ccn1</smiles>

2d<smiles>Cc1ccc(F)c(C(F)(F)F)c1</smiles>

$2 e$<smiles>Cc1ccc([N+](=O)[O-])cc1</smiles><smiles>[R]c1cccc([N+](=O)[O-])c1</smiles>

$2 \mathrm{~g}$<smiles>[Y6]c1ccc(Cl)c([N+](=O)[O-])c1</smiles>

$2 h$<smiles>Cc1ccc([N+](=O)[O-])cc1C</smiles>

2i<smiles>Cc1ccc2c(c1)OCCO2</smiles>

2j<smiles>Cc1ccc(C(F)(F)F)cc1F</smiles>

$\mathbf{R}$

2k<smiles>Cc1cc(-c2ccccc2)[nH]n1</smiles>

2I<smiles>Cc1ccc(Nc2ccccc2)cc1</smiles>

$2 \mathrm{~m}$<smiles>Cc1ccc(C(N)=O)cc1</smiles>

$2 q \quad \mathrm{OCH}_{3}$<smiles>CC(=O)c1cc(C)c(OC(C)(C)C)cc1O</smiles>

2n<smiles>CC(=O)c1ccc(C)cc1</smiles>

20<smiles>C[14c]1ccc(C#N)cc1</smiles>

Scheme 1 Synthesis of aromatic hydrazones $2 \mathrm{a}-2 \mathrm{q}$. Reaction conditions and reagents: (i) $\mathrm{HCl}, \mathrm{NaNO}_{2}, 0^{\circ} \mathrm{C}, 1 \mathrm{~h} ;(\mathrm{ii}) \mathrm{CH}_{2}\left(\mathrm{CN}_{2}, \mathrm{CH}_{3} \mathrm{COONa}, 0{ }^{\circ} \mathrm{C}\right.$, $2 \mathrm{~h}$. 
Table 1 MIC $(\mu \mathrm{M})^{a}$ of aromatic hydrazones against Gram-positive bacteria

\begin{tabular}{lll}
\hline Compounds $^{b}$ & SA & MRSA \\
\hline $\mathbf{2 a}$ & 3.12 & 6.25 \\
$\mathbf{2 b}$ & $>200$ & $>200$ \\
$\mathbf{2 c}$ & 50 & 100 \\
$\mathbf{2 d}$ & 100 & 100 \\
$\mathbf{2 e}$ & 1.56 & 1.56 \\
$\mathbf{2 f}$ & 25 & 50 \\
$2 \mathbf{g}$ & 100 & 100 \\
$\mathbf{2 h}$ & 6.25 & 12.50 \\
$2 \mathbf{i}$ & 50 & 100 \\
$2 \mathbf{j}$ & 1.56 & 1.56 \\
$\mathbf{2 k}$ & $>200$ & $>200$ \\
$\mathbf{2 l}$ & 200 & $>200$ \\
$\mathbf{2 m}$ & 200 & $>200$ \\
$\mathbf{2 n}$ & 25 & 100 \\
$\mathbf{2 0}$ & 100 & 100 \\
$2 \mathbf{p}$ & 50 & 100 \\
$\mathbf{2 q}$ & 100 & 50 \\
$\mathbf{A}$ & 25 & 100 \\
$\mathbf{B}$ & 0.63 & 1.25 \\
$\mathbf{C}$ & 7.50 & 7.50
\end{tabular}

${ }^{a}$ MICs representing mean values of at least three replicates. ${ }^{b} \mathbf{A}$ : cefoxitin, B: ofloxacin, C: linezolid.

aureus (MRSA); two Gram-negative bacteria: Pseudomonas aeruginosa ATCC 9027 (PA) and Escherichia coli ATCC 8739 (EC); and Candida albicans ATCC 10231 (CA), respectively. The results showed that compounds $\mathbf{2 a - 2 q}$ showed no anti-microbial activity against Gram-negative bacteria and fungi (MICs $>200$ $\mu \mathrm{M}$, except for $2 \mathrm{a}, \mathrm{MIC}=50 \mu \mathrm{M}$ for fungi), while some of compounds exhibited the moderate to high level of antibacterial activity against Gram-positive bacteria (Table 1). Amongst them, compounds $2 \mathbf{e}$ and $2 \mathbf{j}$ showed better activity than CCCP (2a) against both $S$. aureus and MRSA (MICs $=1.56 \mu \mathrm{M})$, which are even better than cefoxitin and linezolid, and similar with the MICs of ofloxacin. The growth inhibition effects of compounds 2a, 2e and $2 \mathbf{j}$ were further investigated against both $S$. aureus and MRSA. The results confirmed that both compounds $2 \mathrm{e}$ and $2 \mathbf{j}$ were able to inhibit the growth of $S$. aureus and MRSA effectively at the MIC or higher concentrations. Once the concentration drops down to half or less of the MICs, they could only slow down the growing rate of $S$. aureus and MRSA during logarithmic period, while the growth could be recovered after being incubated for longer time (Fig. 2).

\subsection{SAR analysis}

The structure-activity relationship (SAR) analysis are as followed: (i) when the aromatic ring of carbonyl cyanide $m$-chlorophenylhydrazone (CCCP) was substituted by heterocycle, the antibacterial activity against Gram-positive bacteria was significantly decreased (such as $2 \mathbf{b}, \mathbf{2 c}$ and $2 \mathbf{k}$ ); (ii) the phenyl ring with strong electron-withdrawing substituent $\left(-\mathrm{NO}_{2}\right.$ and $\left.-\mathrm{CF}_{3}\right)$ showed the moderate antibacterial activity (such as $\mathbf{2 d - 2 h}, \mathbf{2 j}$ and 2p); (iii) further, para-substituted group ( $-\mathrm{NO}_{2}$ and $\left.-\mathrm{CF}_{3}\right)$ exhibited better activity than meta-substituted group, e.g. for $S$. aureus and MRSA (MIC values), 2e (1.56, $1.56 \mu \mathrm{M}), 2 \mathrm{~h}(6.25,12.5$ $\mu \mathrm{M})>2 \mathrm{f}(25,100 \mu \mathrm{M}), 2 \mathrm{~g}(100,100 \mu \mathrm{M}) ; 2 \mathrm{j}(1.56,1.56 \mu \mathrm{M})>2 \mathrm{~d}$ $(100,100 \mu \mathrm{M}), 2 \mathbf{p}(50,100 \mu \mathrm{M})$.

\subsection{Cytotoxicity assays}

The human hepatic L02 cells were treated with different concentrations of tested compound $(3.125,6.25,12.5,25,50$
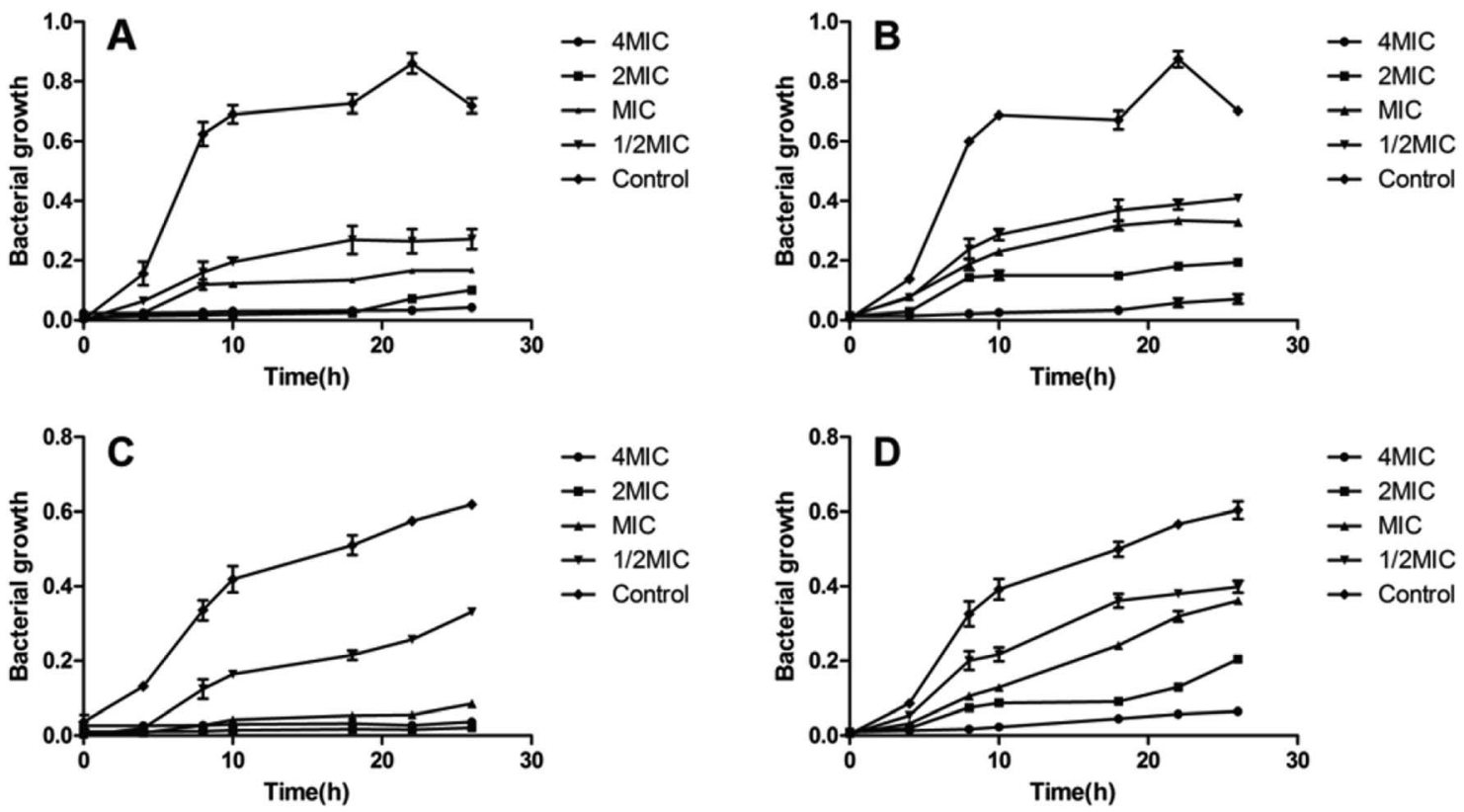

Fig. 2 S. aureus and MRSA growth inhibition curves. Titration curves showing the effect of different concentrations of compounds $2 e$ and $2 j$ on the growth of S. aureus ( $A$ and B) and MRSA ( $C$ and D). Each OD point presented is the average values of three tests and all experiments are internally controlled. Data are presented as the mean \pm standard deviation $(n=3)$. 


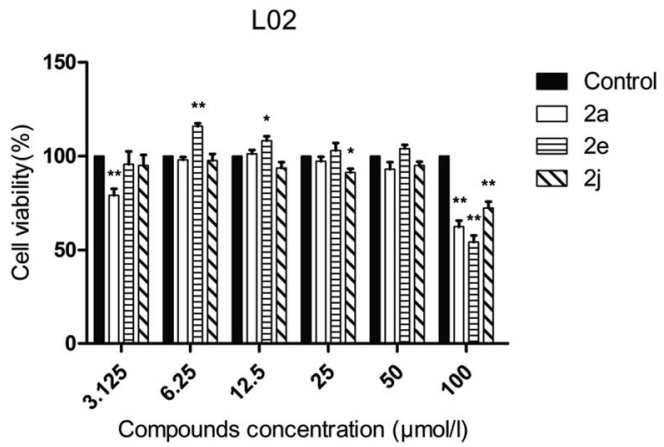

Fig. 3 Cell viability assay of tested compounds to L02 cells. Data are presented as the mean \pm standard error $(n=3)$, one-way ANOVA (vs. control), $* P<0.05, * * P<0.01$. and $100 \mu \mathrm{M}$ ), and cell viability was measured after $24 \mathrm{~h}$ using MTT method. As shown in Fig. 3, compounds 2a, 2e and $2 \mathbf{j}$ at the test concentrations $(3.125-50 \mu \mathrm{M})$ had no obvious cytotoxicity against L02 cells, and the relative cell viabilities of treated cells were all more than $70 \%$.

\subsection{Checkerboard assay}

To develop a feasible medical application, active compounds $2 \mathbf{a}$, $2 \mathbf{e}$ and $\mathbf{2} \mathbf{j}$ were tested in combination with clinical antibiotics on SA and MRSA by checkerboard assay in order to evaluate their ability to improve the anti-bacterial activity. ${ }^{20}$ Each checkerboard test generates many different combinations and, by convention, the FIC value of the most effective combination was used in calculating the fractional inhibitory concentration index (FICI). FICI was calculated by adding both FICs:

Table 2 Synergistic activity assays on MRSA

\begin{tabular}{|c|c|c|c|c|c|c|c|}
\hline Compounds & $\overline{\mathbf{B}}$ & $\mathbf{A}$ & B & $\mathbf{A}$ & B & FICI & Mode of action \\
\hline $2 \mathbf{e}+$ ofloxacin & & 1.56 & 1.25 & 0.39 & 0.04 & 0.28 & Synergy \\
\hline $2 \mathbf{j}+$ ofloxacin & & 1.56 & 1.25 & 0.78 & 0.04 & 0.53 & Additive \\
\hline $2 a+$ cefoxitin & & 6.25 & 50.00 & 3.12 & 1.56 & 0.53 & Additive \\
\hline $2 a+$ linezolid & & 6.25 & 7.50 & 3.12 & 0.47 & 0.56 & Additive \\
\hline $2 \mathbf{e}+$ linezolid & & 1.56 & 7.50 & 0.78 & 0.47 & 0.56 & Additive \\
\hline $2 \mathbf{j}+$ linezolid & & 1.56 & 7.50 & 0.78 & 1.90 & 0.75 & Additive \\
\hline
\end{tabular}

A

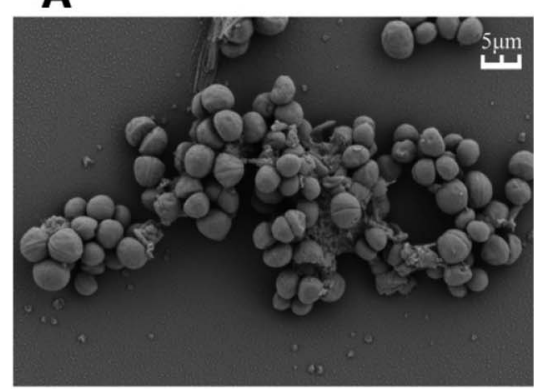

D

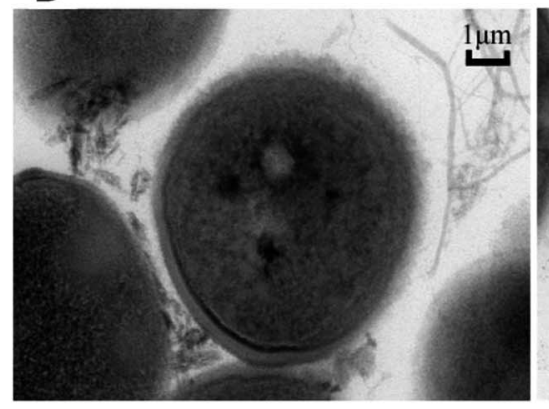

B

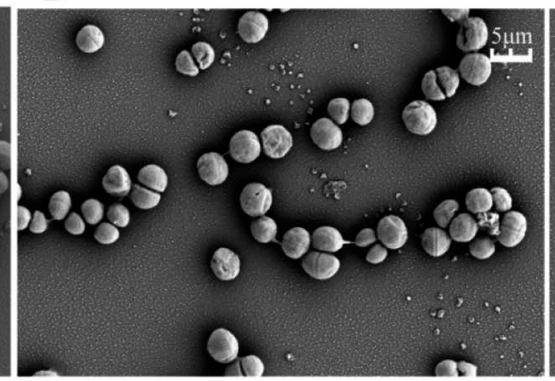

$\mathbf{E}$

\section{C}

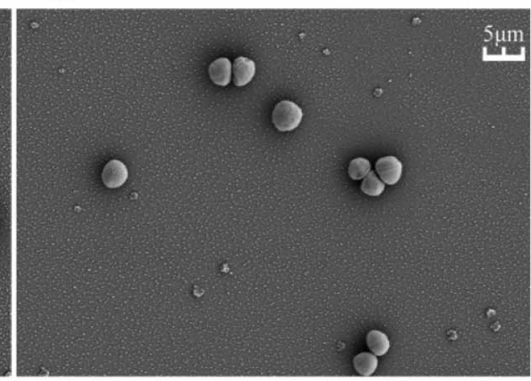

$\mathbf{F}$
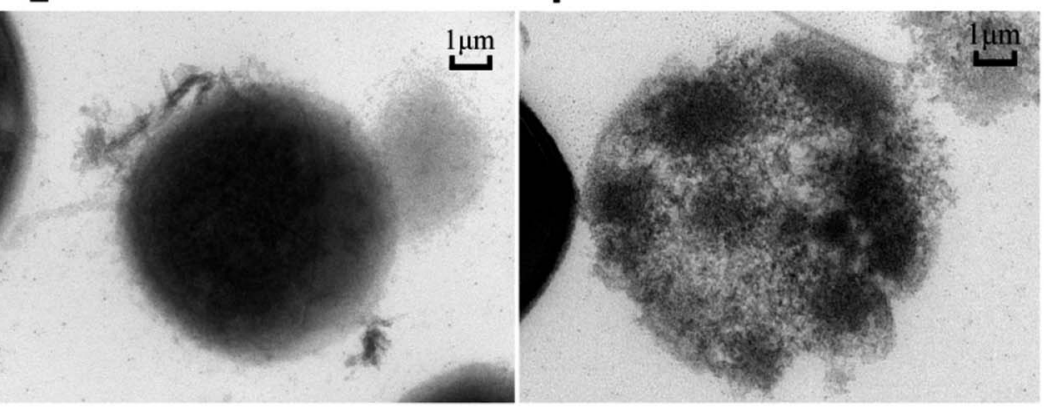

Fig. 4 Electronic microscopies of MRSA (A-C). Scanning electronic microscopic images of MRSA and (D-F) Transmission electronic microscopic images of MRSA. (A and D) represent untreated bacteria. ( $\mathrm{B}$ and $\mathrm{E}$ ) represent bacteria treated with compound $2 \mathrm{e}$ at $1 / 2 \mathrm{MIC}$. (C and F) represent bacteria treated with compound $2 \mathrm{e}$ at $1 / 2 \mathrm{MIC}$ and ofloxacin of 1/8 MIC. 


$$
\mathrm{FICI}=\mathrm{FIC}_{\mathbf{A}}+\mathrm{FIC}_{\mathbf{B}}=\mathrm{C}_{\mathbf{A}}^{\text {comb }} / \mathrm{MIC}_{\mathbf{A}}^{\text {alone }}+\mathrm{C}_{\mathbf{B}}^{\text {comb }} / \mathrm{MIC}_{\mathbf{B}}^{\text {alone }}
$$

where $\mathbf{M I C}_{\mathbf{A}}^{\text {alone }}$ and MIC $_{\mathbf{B}}^{\text {alone }}$ are the MICs of compound $\mathbf{A}$ and $\mathbf{B}$ when acting alone and $\mathbf{C}_{\mathbf{A}}^{\text {comb }}$ and $\mathbf{C}_{\mathbf{B}}^{\text {comb }}$ are concentrations of compounds $\mathbf{A}$ and $\mathbf{B}$ at the isoeffective combinations. The FICI was interpreted as synergistic when it was $\leq 0.5$, additional effects when $0.5<$ FICI $\leq 1.0$, indifferent when $1.0<$ FICI $\leq 2.0$, and antagonistic when FICI $>2.0$, and any value between was interpreted as indifferent.

As shown in Table 2, when compound 2 a was used in combination with antibiotics, MIC was reduced 2-fold, but for compound 2e, MIC was reduced 4-fold. Moreover, compounds $\mathbf{2 a}, 2 \mathbf{e}$ and $\mathbf{2 j}$ could significantly improve the performance of clinical antibiotics, for example, ofloxacin, cefoxitin and linezolid lowered their MIC values from 1.25, 50.0 and $7.5 \mu \mathrm{M}$ to $0.04,1.56$ and $0.47 \mu \mathrm{M}$, respectively. Calculations of FIC and FICI (always less than 1.0) obtained by checkerboard assays on SA and MRSA showed at least additive effects of active compounds (2a, 2e and $2 \mathbf{j}$ ) with clinical antibiotics. When compound 2e was combined with ofloxacin, FICI value of 0.28 suggested a synergistic effect. Therefore, worthy of note is the prophylactic purpose that low doses of clinical antibiotics plus a protonophore may be developed as an anti-MRSA therapy by inhibiting biofilm.

\subsection{Electron microscope}

To elucidate the effects of compound 2e on MRSA, both the Scanning Electron Microscopy (SEM) and Transmission Electron Microscopy (TEM) were used to observe the bacteria after being treated with either compound 2e alone or in combination with ofloxacin. As shown in Fig. 4, SEM results revealed that untreated MRSA form biofilms in normal growth condition, while the biofilms were eradicated when treated with compound $2 \mathrm{e}$ at $1 / 2$ MIC concentration or in its combination with 1/8 MIC of ofloxacin. Furthermore, TEM results revealed that the regular cell conformation was destructed and the leakage of cellular substances under the treatment of compound $2 \mathrm{e}$ in combination with ofloxacin.

\section{Conclusion}

In summary, a series of aromatic hydrazones were synthesized and evaluated for their antibacterial activities. Some compounds showed potential antimicrobial activity against Gram-positive bacteria, amongst them, compounds $2 \mathbf{e}$ and $2 \mathbf{j}$ had the lowest MICs against both $S$. aureus and MRSA $(1.56 \mu \mathrm{M})$, and the growth inhibition assay confirmed the inhibition effects. SAR showed that (i) for aromatic hydrazones containing heterocycles, the antibacterial activity against Gram-positive bacteria was significantly decreased $(2 \mathbf{a}>\mathbf{2 b}, \mathbf{2 c}, \mathbf{2 k})$; (ii) the phenyl ring with strong electron-withdrawing substituent $\left(-\mathrm{NO}_{2}\right.$ and $\left.-\mathrm{CF}_{3}\right)$ showed the moderate antibacterial activity $(\mathbf{2 d - 2 h}, \mathbf{2 j}$ and 2p); (iii) further, para-substituted group $\left(-\mathrm{NO}_{2}\right.$ and $\left.-\mathrm{CF}_{3}\right)$ exhibited better activity than meta-substituted group, e.g. for $S$. aureus and MRSA (MIC values), 2e, 2h > 2f, 2g; 2j > 2d, 2p. Aromatic hydrazones in combination with clinical antibiotics exhibited better Gram-positive antibacterial activities, especially when compound $2 \mathrm{e}$ was used in combination with ofloxacin, in which the synergistic effect was observed. MTT assay showed that the toxicity of compound $2 \mathrm{e}$ was low as that of CCCP. Further, electron microscopy showed that compound $2 \mathrm{e}$ possesses the capability to inhibit the formation of biofilm and eradicate already existing biofilm. In sum, compound 2e displayed antibacterial activity against MRSA through inhibiting biofilm, especially improved the bactericidal effects of clinical antibiotics by synergistic effect. Therefore, the aromatic residue is a promising scaffold for further antibacterial modifications.

\section{Materials and methods}

\subsection{Chemistry}

All reagents were purchased from commercial sources and were used without further purification. Melting points (uncorrected) were determined on a XT4MP apparatus (Taike Corp., Beijing, China). ${ }^{1} \mathrm{H}$ NMR and ${ }^{13} \mathrm{C}$ NMR spectra were recorded on Bruker $\mathrm{AV}-600$ or AV-400 MHz instruments in $\mathrm{CDCl}_{3}$. Chemical shifts are reported in parts per million $(\delta)$ downfield from the signal of tetramethylsilane (TMS) as internal standards. Coupling constants are reported in Hz. The multiplicity is defined by $\mathrm{s}$ (singlet), d (doublet), t (triplet), or m (multiplet). High resolution mass spectra (HRMS) were obtained on an Agilent 12606221 TOF mass spectrometry. Column and thin-layer chromatography (CC and TLC, resp.) were performed on silica gel (200300 mesh) and silica gel $\mathrm{GF}_{254}$ (Qingdao Marine Chemical Factory) respectively.

\subsection{General procedures for synthesis of 2-(2-arylhydrazono) malononitriles $2 \mathrm{a}-2 \mathrm{q}$}

To a solution of the aromatic amine $(15 \mathrm{mmol})$ and concentrated $\mathrm{HCl}(37 \%, 13.8 \mathrm{~mL})$ in $\mathrm{H}_{2} \mathrm{O}(75 \mathrm{~mL})$ was dropwise added $\mathrm{NaNO}_{2}(15 \mathrm{mmol} 1.04 \mathrm{~g})$ in $\mathrm{H}_{2} \mathrm{O}(50 \mathrm{~mL})$ for $1 \mathrm{~h}$ in an ice bath, and the mixture was stirred for $30 \mathrm{~min}$. Then, the reaction solution was added to a solution of $\mathrm{CH}_{2}(\mathrm{CN})_{2}(20 \mathrm{mmol}, 1.26$ $\mathrm{mL}$ ) and NaOAc (31 mmol, $38.1 \mathrm{~g})$ in $\mathrm{H}_{2} \mathrm{O}(130 \mathrm{~mL})$ under continuous stirring at $0{ }^{\circ} \mathrm{C}$. After 2 hours, the reaction mixture was filtrated, washed twice with water, and the residue was recrystallized from ethanol to give the title compounds $\mathbf{2 a - 2 q}$.

$\boldsymbol{N}$-(3-Chlorophenyl)carbonohydrazonoyl dicyanide (2a). Yellow-green powder, yield, 80\%; mp 131-133 ${ }^{\circ} \mathrm{C} ;{ }^{1} \mathrm{H}$ NMR (400 MHz, DMSO- $\left.d_{6}\right) \delta 7.49-7.40(\mathrm{~m}, 3 \mathrm{H}), 7.26(\mathrm{~d}, J=6.9 \mathrm{~Hz}, 1 \mathrm{H}) .{ }^{13} \mathrm{C}$ NMR (151 MHz, $\left.\mathrm{CDCl}_{3}\right) \delta 142.71,135.01,130.30,125.54,116.69$, 114.73, 113.44, 109.53, 85.54.

$\boldsymbol{N}$-(2-Chloropyridin-4-yl)carbonohydrazonoyl dicyanide (2b). Yellow powder, yield, 83\%; mp 92-94 ${ }^{\circ} \mathrm{C} ;{ }^{1} \mathrm{H}$ NMR $(400 \mathrm{MHz}$, DMSO- $\left.d_{6}\right) \delta 8.25(\mathrm{~d}, J=5.3 \mathrm{~Hz}, 1 \mathrm{H}), 7.29(\mathrm{~d}, J=5.1 \mathrm{~Hz}, 2 \mathrm{H}) .{ }^{13} \mathrm{C}$ NMR (151 MHz, $\left.\mathrm{CDCl}_{3}\right) \delta 153.60,151.86,149.81,114.60,111.33$, 110.86, 110.60, 86.25. TOF-HRMS: $m / z[\mathrm{M}+\mathrm{H}]^{+}$calcd for $\mathrm{C}_{8} \mathrm{H}_{4} \mathrm{ClN}_{5}$ : 206.0155; found: 206.0156.

$\mathrm{N}$-(2-Methoxypyridin-4-yl)carbonohydrazonoyl dicyanide (2c). Orange powder, yield, 78\%; mp 42-43 ${ }^{\circ} \mathrm{C} ;{ }^{1} \mathrm{H}$ NMR (400 $\left.\mathrm{MHz} \mathrm{CDCl}_{3}\right) \delta 8.17(\mathrm{~d}, J=5.8 \mathrm{~Hz}, 1 \mathrm{H}), 6.90(\mathrm{dd}, J=5.7,1.7 \mathrm{~Hz}$, $1 \mathrm{H}), 6.67(\mathrm{~s}, 1 \mathrm{H}), 3.97(\mathrm{~s}, 3 \mathrm{H}) .{ }^{13} \mathrm{C}$ NMR (151 MHz, DMSO- $\left.d_{6}\right)$ 
$\delta$ 163.06, 115.16, 110.59, 106.22 (2C), 95.33 (2C), 87.73, 54.98. TOF-HRMS: $m / z[\mathrm{M}+\mathrm{H}]^{+}$calcd for $\mathrm{C}_{9} \mathrm{H}_{7} \mathrm{~N}_{5} \mathrm{O}$ : 202.0651; found: 202.0649 .

$\mathbf{N}$-(4-Fluoro-3-(trifluoromethyl)phenyl)carbonohydrazonoyl dicyanide (2d). Earthy red powder, yield, 78\%; mp $30{ }^{\circ} \mathrm{C} ;{ }^{1} \mathrm{H}$ NMR (400 MHz, $\left.\mathrm{CDCl}_{3}\right) \delta 10.06(\mathrm{~s}, 1 \mathrm{H}), 7.82-7.42(\mathrm{~m}, 2 \mathrm{H}), 7.31$ $(\mathrm{t}, J=9.1 \mathrm{~Hz}, 1 \mathrm{H}) .{ }^{13} \mathrm{C}$ NMR $\left(101 \mathrm{MHz}, \mathrm{DMSO}-d_{6}\right) \delta 157.75$, $138.80,122.89,122.78,119.27,117.72$, 115.36, 114.46, 110.15, 86.63 .

$\mathrm{N}$-(4-Nitrophenyl)carbonohydrazonoyl dicyanide (2e). Bright yellow powder, yield, 81\%; mp $40-41{ }^{\circ} \mathrm{C} ;{ }^{1} \mathrm{H}$ NMR $(400 \mathrm{MHz}$, DMSO- $\left.d_{6}\right) \delta 8.19(\mathrm{~d}, J=9.1 \mathrm{~Hz}, 2 \mathrm{H}), 7.49(\mathrm{~d}, J=9.1 \mathrm{~Hz}, 2 \mathrm{H}) .{ }^{13} \mathrm{C}$ NMR (101 MHz, DMSO- $d_{6}$ ) $\delta$ 142.71, 125.19 (2C), 118.14 (2C), 117.98, 113.20, 94.76, 82.41. TOF-HRMS: $m / z[\mathrm{M}+\mathrm{H}]^{+}$calcd for $\mathrm{C}_{9} \mathrm{H}_{6} \mathrm{~N}_{5} \mathrm{O}_{2}$ : 230.0547; found: 230.0550 .

$\mathrm{N}$-(3-Nitrophenyl)carbonohydrazonoyl dicyanide (2f). Yellow powder, yield, 82\%; mp 144-145 ${ }^{\circ} \mathrm{C} ;{ }^{1} \mathrm{H}$ NMR (400 MHz, DMSO$\left.d_{6}\right) \delta 8.21(\mathrm{t}, J=2.1 \mathrm{~Hz}, 1 \mathrm{H}), 8.08-7.96(\mathrm{~m}, 1 \mathrm{H}), 7.91-7.77(\mathrm{~m}$, $1 \mathrm{H}), 7.68(\mathrm{t}, J=8.2 \mathrm{~Hz}, 1 \mathrm{H}) .{ }^{13} \mathrm{C}$ NMR (151 MHz, DMSO- $\left.d_{6}\right)$ $\delta 148.91,144.78,131.33,123.26,119.80,115.30,111.59,110.85$, 85.83.

$\mathrm{N}$-(4-Chloro-3-nitrophenyl)carbonohydrazonoyl dicyanide (2g). Yellow powder, yield, 80\%; mp 40-41 ${ }^{\circ} \mathrm{C} ;{ }^{1} \mathrm{H}$ NMR (400 MHz, DMSO- $\left.d_{6}\right) \delta 7.73(\mathrm{~s}, 1 \mathrm{H}), 7.56(\mathrm{~d}, J=8.7 \mathrm{~Hz}, 1 \mathrm{H}), 7.48(\mathrm{~d}, J$ $=8.7 \mathrm{~Hz}, 1 \mathrm{H}) .{ }^{13} \mathrm{C}$ NMR $\left(101 \mathrm{MHz}, \mathrm{DMSO}-d_{6}\right) \delta 158.90,153.42$, 136.88, 129.41, 126.08, 122.79, 121.01, 119.43, 81.46.

$\mathrm{N}$-(2-Methyl-4-nitrophenyl)carbonohydrazonoyl dicyanide (2h). Dark green powder, yield, 85\%; mp $41{ }^{\circ} \mathrm{C} ;{ }^{1} \mathrm{H}$ NMR $(400$ $\left.\mathrm{MHz} \mathrm{CDCl}_{3}\right) \delta 9.57(\mathrm{~s}, 1 \mathrm{H}), 8.18(\mathrm{dt}, J=27.6,12.5 \mathrm{~Hz}, 2 \mathrm{H}), 7.70$ $(\mathrm{d}, J=8.9 \mathrm{~Hz}, 1 \mathrm{H}), 2.51(\mathrm{~s}, 3 \mathrm{H}) .{ }^{13} \mathrm{C} \mathrm{NMR}\left(101 \mathrm{MHz}, \mathrm{CDCl}_{3}\right)$ $\delta 146.56,144.00,129.46,126.58,122.61,118.70,114.81,110.23$, $87.24,17.47$.

$\mathrm{N}$-(2,3-Dihydrobenzo[ $[\mathrm{b}][1,4]$ dioxin-6-yl)carbonohydrazonoyl dicyanide (2i). Earth orange powder, yield, 84\%; mp $90{ }^{\circ} \mathrm{C} ;{ }^{1} \mathrm{H}$ NMR (400 MHz, DMSO- $\left.d_{6}\right) \delta$ 6.99-6.94 (m, 1H), 6.92-6.87 (m, $1 \mathrm{H}), 4.25(\mathrm{~s}, 4 \mathrm{H}) .{ }^{13} \mathrm{C}$ NMR (101 MHz, DMSO- $\left.d_{6}\right) \delta$ 144.21, $142.25,136.12$, 118.20, 115.24, 110.81, 110.17, 105.94, 83.35, $64.68,64.49$.

$\mathrm{N}$-(2-Fluoro-5-(trifluoromethyl)phenyl)carbonohydrazonoyl dicyanide (2j). Orange powder, yield, 90\%; mp $33-35{ }^{\circ} \mathrm{C} ;{ }^{1} \mathrm{H}$ NMR (400 MHz, DMSO- $\left.d_{6}\right) \delta 7.80(\mathrm{~d}, J=6.9 \mathrm{~Hz}, 1 \mathrm{H}), 7.68-7.51$ $(\mathrm{m}, 2 \mathrm{H}) .{ }^{13} \mathrm{C}$ NMR $\left(101 \mathrm{MHz}, \mathrm{DMSO}-d_{6}\right) \delta 155.03,132.74,126.42$, $124.09,124.06,118.49,117.50,115.37,110.80$, 87.05. TOFHRMS: $m / z[\mathrm{M}+\mathrm{Na}]^{+}$calcd for $\mathrm{C}_{10} \mathrm{H}_{4} \mathrm{~F}_{4} \mathrm{~N}_{4} \mathrm{Na}$ : 279.0264; found: 279.0261 .

$\mathrm{N}$-(5-Phenyl-1H-pyrazol-3-yl)carbonohydrazonoyl dicyanide (2k). Yellow powder, yield, 89\%; mp $138-139{ }^{\circ} \mathrm{C} ;{ }^{1} \mathrm{H}$ NMR $(400$ MHz, DMSO- $\left.d_{6}\right) \delta 9.33(\mathrm{~s}, 2 \mathrm{H}), 8.17(\mathrm{~d}, J=7.5 \mathrm{~Hz}, 2 \mathrm{H}), 7.66(\mathrm{~s}$, $1 \mathrm{H}), 7.53$ (dt, $J=13.8,7.1 \mathrm{~Hz}, 3 \mathrm{H}) .{ }^{13} \mathrm{C}$ NMR (101 MHz, DMSO$\left.d_{6}\right) \delta 156.98,150.06,143.24,131.62,130.41,129.41(2 \mathrm{C}), 127.14$ (2C), 116.37, 105.62, 96.13. TOF-HRMS: $m / z[\mathrm{M}+\mathrm{H}]^{+}$calcd for $\mathrm{C}_{12} \mathrm{H}_{8} \mathrm{~N}_{6}$ : 237.0801; found: 237.0805 .

$\mathrm{N}$-(4-Acetamidophenyl)carbonohydrazonoyl dicyanide (2l). Yellow powder, yield, 70\%; mp 45-46 ${ }^{\circ} \mathrm{C} ;{ }^{1} \mathrm{H}$ NMR $(400 \mathrm{MHz}$, DMSO- $\left.d_{6}\right) \delta 10.07(\mathrm{~s}, 1 \mathrm{H}), 7.63(\mathrm{~d}, J=8.8 \mathrm{~Hz}, 2 \mathrm{H}), 7.40(\mathrm{~d}, J=8.8$ $\mathrm{Hz}, 2 \mathrm{H}), 2.05$ (s, 3H). ${ }^{13} \mathrm{C}$ NMR (101 MHz, DMSO- $\left.d_{6}\right) \delta 168.77$,
137.82, 137.56, 120.18 (2C), 117.56 (2C), 115.42, 110.94, 83.40, 24.43.

$\boldsymbol{N}$-(4-Carbamoylphenyl)carbonohydrazonoyl dicyanide (2m). Dark yellow green powder, yield, 82\%; mp 155-157 ${ }^{\circ} \mathrm{C} ;{ }^{1} \mathrm{H}$ NMR (400 MHz, DMSO- $\left.d_{6}\right) \delta 7.96(\mathrm{~s}, 1 \mathrm{H}), 7.90(\mathrm{~d}, J=8.8 \mathrm{~Hz}, 2 \mathrm{H}), 7.46$ $(\mathrm{d}, J=8.8 \mathrm{~Hz}, 2 \mathrm{H}), 7.33(\mathrm{~s}, 1 \mathrm{H}) .{ }^{13} \mathrm{C}$ NMR (101 MHz, DMSO- $\left.d_{6}\right)$ $\delta$ 167.69, 146.72, 131.09 (2C), 129.38 (2C), 116.90, 116.29, 111.71, 84.09.

$\mathrm{N}$-(4-Acetylphenyl)carbonohydrazonoyl dicyanide (2n). Bright yellow powder, yield, 91\%; mp 254-255 ${ }^{\circ} \mathrm{C} ;{ }^{1} \mathrm{H}$ NMR (400 MHz, DMSO- $\left.d_{6}\right) \delta 7.93(\mathrm{~d}, J=8.6 \mathrm{~Hz}, 1 \mathrm{H}), 7.46(\mathrm{~d}, J=8.6 \mathrm{~Hz}$, $1 \mathrm{H}), 2.51(\mathrm{~s}, 1 \mathrm{H}) .{ }^{13} \mathrm{C}$ NMR (101 MHz, DMSO- $\left.d_{6}\right) \delta$ 197.03, 150.90, 133.24, 130.23 (2C), 117.73 (2C), 113.00, 99.98, 82.71, 26.99.

$\boldsymbol{N}$-(4-Cyanophenyl)carbonohydrazonoyl dicyanide (20). Yellow-green powder, yield, 88\%; mp $116-117{ }^{\circ} \mathrm{C} ;{ }^{1} \mathrm{H}$ NMR (400 MHz, DMSO- $\left.d_{6}\right) \delta 7.78(\mathrm{~d}, J=8.4 \mathrm{~Hz}, 2 \mathrm{H}), 7.51(\mathrm{~d}, J=8.4 \mathrm{~Hz}$, $2 \mathrm{H}) .{ }^{13} \mathrm{C}$ NMR (101 MHz, DMSO- $\left.d_{6}\right) \delta 149.82,134.03$ (2C), 119.57, 118.29 (2C), 116.88, 112.26, 106.62, 84.34.

$\mathrm{N}$-(3,5-Bis(trifluoromethyl)phenyl)carbonohydrazonoyl dicyanide (2p). Light yellow powder, yield, 70\%; mp $113-115{ }^{\circ} \mathrm{C}$; ${ }^{1} \mathrm{H}$ NMR (400 MHz, $\left.\mathrm{CDCl}_{3}\right) \delta 7.86(\mathrm{~s}, 2 \mathrm{H}), 7.73(\mathrm{~s}, 1 \mathrm{H})$.

$\mathrm{N}$-(5-Acetyl-4-hydroxy-2-methoxyphenyl)carbonohydrazonoyl dicyanide (2q). Dark green powder, yield, 70\%; mp 132-134 ${ }^{\circ} \mathrm{C}$; ${ }^{1} \mathrm{H}$ NMR (400 MHz, DMSO- $d_{6}$ ) $\delta 12.58(\mathrm{~s}, 1 \mathrm{H}), 7.79$ (s, 1H), 6.69 $(\mathrm{s}, 1 \mathrm{H}), 3.95(\mathrm{~s}, 3 \mathrm{H}), 2.59(\mathrm{~s}, 3 \mathrm{H}) .{ }^{13} \mathrm{C}$ NMR (101 MHz, DMSO- $\left.d_{6}\right)$ $\delta$ 203.23, 162.86, 156.87, 123.39, 122.67, 114.81, 113.71, 110.20, 100.94, 85.12, 57.21, 27.67.

\subsection{Culture conditions and treatments}

L02 (normal human liver) cell lines were purchased from the Russian Cell Culture Collection (Institute of Cytology Russian Academy of Science, Saint Petersburg, Russia). L02 cells were maintained in Dulbecco's modified Eagle's medium (DMEM) (Invitrogen, USA) supplemented with 2 mM L-glutamine (SigmaAldrich, UK), 10\% fetal bovine serum (Invitrogen, USA), $50 \mu \mathrm{g}$ $\mathrm{mL}^{-1}$ gentamicin sulfate (Invitrogen, USA) at $37{ }^{\circ} \mathrm{C}$ and $5 \% \mathrm{CO}_{2}$. All compounds were dissolved in 100\% DMSO (Sigma-Aldrich, UK) to $100 \mathrm{mM}$ stock solutions and diluted in completed DMEM immediately before addition to the assay plates. DMSO was maintained at a final concentration of $0.1 \%$.

\subsection{Minimum inhibitory concentrations (MICs)}

The MICs of tested compounds were determined using Mueller-Hinton (MH) broth micro-broth dilution assay established by the Clinical Laboratory Standards Institute (CLSI) in 96-well micro-test plates. The final test concentration ranged from 0.39 to $200 \mu \mathrm{M}$ and the bacterial inocula was $10^{8} \mathrm{CFU} \mathrm{mL}^{-1}$. After 1820 hours of incubation at $37^{\circ} \mathrm{C}$, the MICs were determined to be the lowest concentration of tested compound that inhibited the apparent increase in microorganisms. Each experiment was repeated at least 3 times to report the MIC value. ${ }^{26}$

\subsection{Inhibition of bacterial growth}

The effect of concentrations ranging from 0.5 to 4 times MIC of the active compounds on the growth of $S$. aureus or MRSA was 
quantified after incubation at $35^{\circ} \mathrm{C}$ for $0,4,8,10,18,22$ and 26 hours. At each time point, an aliquot $(100 \mu \mathrm{L})$ was pipetted and measured for the $A_{450} \mathrm{~nm}$. The experiment was performed in three biologically independent assays, each tested in triplicate.

\subsection{Checkerboard assays}

The synergistic effect of the combination of clinical antibacterials with the tested compounds was determined by checkerboard microdilution assays. In brief, checkerboards were set up with double dilutions of compounds $2 \mathrm{a}(0-12.5 \mu \mathrm{M})$ or $2 \mathrm{e}(0-$ $3.12 \mu \mathrm{M})$ or $2 \mathrm{j}(0-3.12 \mu \mathrm{M})$ in the horizontal wells and ofloxacin $(0-2.5 \mu \mathrm{M})$ or cefoxitin $(0-100 \mu \mathrm{M})$ or linezolid $(0-14 \mu \mathrm{M})$ in the vertical wells. Then $50 \mu \mathrm{L}$ each was arranged on the rows and columns of the plate, and $100 \mu \mathrm{L}$ of MRSA was added to the

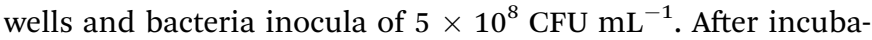
tion at $35{ }^{\circ} \mathrm{C}$ for 20 hours in 96-well micro-test plates. Aromatic hydrazones were further tested to determine their nature of interaction (synergy, antagonism, additive or no interaction) with ofloxacin, cefoxitin and linezolid and expressed as the fractional inhibitory concentration index (FICI) for each agent.

\subsection{Cell viability}

Cell viability was performed against L02 (normal human liver cell line) cells using the MTT assay. L02 cells were grown in DMEM containing $10 \%$ fetal calf serum, 100 units per $\mathrm{mL}$ penicillin and $100 \mu \mathrm{g} \mathrm{mL}{ }^{-1}$ streptomycin at $37^{\circ} \mathrm{C}$ in a $5 \% \mathrm{CO}_{2}$ incubator. L02 cells were seeded at $1 \times 10^{4}$ cells per well in 96well micro-test plates. After $24 \mathrm{~h}$ of culture, the cells were treated with different concentrations of tested compound. After $24 \mathrm{~h}, 20 \mu \mathrm{L}$ of $0.5 \mathrm{mg} \mathrm{mL}{ }^{-1}$ MTT reagent was added to the cells and incubated for $4 \mathrm{~h}$. After $4 \mathrm{~h}$, the liquid in the well was discarded, and then $150 \mu \mathrm{L}$ of DMSO was added to dissolve the formazan. The absorbance value $\left(\mathrm{OD}_{570}\right)$ was measured at 570 $\mathrm{nm}$. The cell percentage survival rate was calculated by setting the density of formazan formed in the blank group to $100 \%$ viability as a control. Cell viability $(\%)=$ compound $\left(\mathrm{OD}_{570}\right) /$ blank $\left(\mathrm{OD}_{570}\right) \times 100 \%$. Each compound was tested in triplicate.

\subsection{Electron microscope}

MRSA (ATCC 43300) was grown overnight at $37^{\circ} \mathrm{C}$ on MuellerHinton Agar. The bacteria were harvested and the OD of bacteria suspended in MHB was adjusted to $\sim 0.5$ MacFarlane units so as to give $5 \times 10^{7} \mathrm{CFU} \mathrm{mL}^{-1}$. Bacteria were then aliquoted into $10 \mathrm{~mL}$ tubes and compound 2e dissolved in DMSO was added to give a final concentration ranging from 0.5 to $4 \mathrm{mg}$ $\mathrm{L}^{-1}$ (two fold serial dilutions). After incubation at $37^{\circ} \mathrm{C}$ for $24 \mathrm{~h}$, the bacteria were harvested by centrifugation at $4000 \mathrm{rpm}$, and cell pellets were then re-suspended with $10 \mathrm{mM}$ PBS, pH 7.2 and harvested at $4000 \mathrm{rpm}$. The bacteria were fixed using $2.5 \%$ glutaraldehyde for $3 \mathrm{~h}$, following by washing with $0.1 \mathrm{M} \mathrm{PBS}(\mathrm{pH}$ 7.2) for three times. The washing buffer was then removed and the bacteria were post-fixed in $1 \% \mathrm{OsO}_{4}$ for $2 \mathrm{~h}$. The $\mathrm{OsO}_{4}$ were then pipetted out into an osmium waste bottle and the bacteria were washed in PBS ( $\mathrm{pH}$ 7.2) for three times. Fixed microbial pellets were processed in graded alcohols, propylene oxide, and araldite and cured for $48 \mathrm{~h}$ at $60^{\circ} \mathrm{C}$. Sample were finally stained with uranyl acetate and lead citrate before examine with Hitachi TEM system at an accelerating voltage of $80 \mathrm{kV}$. The SEM model used is the Hitachi su8100 at $3.0 \mathrm{kV}$ voltage. ${ }^{27}$

\subsection{Statistical analysis}

All results were expressed as mean values \pm standard deviation. One-way analysis of variance followed by Dunnett's post hoc test was used for all comparisons.

\section{Conflicts of interest}

There are no conflicts of interest to declare.

\section{Acknowledgements}

Financial support was provided by the Natural Science Foundation of Anhui provincial Department of Education (KJ2019ZD21, KJ2018B12), and Provincial Natural Science Foundation of Anhui (2008085MH261).

\section{References}

1 K. M. G. O'Connell, J. T. Hodgkinson, H. F. Sore, M. Welch, G. P. C. Salmond and D. R. Spring, Angew. Chem., Int. Ed., 2013, 52(41), 10706-10733.

2 R. Tommasi, D. G. Brown, G. K. Walkup, J. I. Manchester and A. A. Miller, Nat. Rev. Drug Discovery, 2015, 14, 529-542.

3 G. D. Wright, Nat. Prod. Rep., 2017, 34(7), 694-701.

4 M. Tyers and G. D. Wright, Nat. Rev. Microbiol., 2019, 17, 141-155.

5 V. Cattoir and B. Felden, J. Infect. Dis., 2019, 220(3), 350-360. 6 S. E. Weinberg, L. A. Sena and N. S. Chandel, Immunity, 2015, 42, 406-417.

7 M. F. Richter, B. S. Drown, A. P. Riley, A. Garcia, T. Shirai, R. L. Svec and P. J. Hergenrother, Nature, 2017, 545(7654), 299-304.

8 B. Z. Cavari and Y. Avi-Dor, Biochem. J., 1967, 103(2), 601608.

9 P. Mishra and D. C. Chan, J. Cell Biol., 2016, 212, 379-387.

10 O. Lomovskaya, M. S. Warren, A. Lee, J. Galazzo, R. Fronko, M. Lee, J. Blais, D. Cho, S. Chamberland, T. Renau, R. Leger, S. Hecker, W. Watkins, K. Hoshino, H. Ishida and V. J. Lee, Antimicrob. Agents Chemother., 2001, 45, 105-116.

11 T. D. Davis, C. J. Gerry and D. S. Tan, ACS Chem. Biol., 2014, 9, 2535-2544.

12 H. Cai, K. Rose, L.-H. Liang, S. Dunham and C. Stover, Anal. Biochem., 2009, 385, 321-325.

13 M. Ghoul, M. Pommepuy, A. Moillo-Batt and M. Cormier, Appl. Environ. Microbiol., 1989, 55, 1040-1043.

14 J. B. Michel, P. J. Yeh, R. Chait, R. C. Moellering Jr and R. Kishony, Proc. Natl. Acad. Sci. U. S. A., 2008, 105(39), 14918-14923.

15 R. C. Allen and S. P. Brown, mBio, 2019, 10(5), e01831-19.

16 N. Tharmalingam, E. Jayamani, R. Rajamuthiah, D. Castillo, B. B. Fuchs, M. J. Kelso and E. Mylonakis, Future Med. Chem., 2017, 9(12), 1401-1411. 
17 J. M. Stokes, C. R. Macnair, B. Ilyas, S. French, J.-P. Côté, C. Bouwman, M. A. Farha, A. O. Sieron, C. Whitfield, B. K. Coombes and E. D. Brown, Nat. Microbiol., 2017, 2, 17028.

18 D. Sinha, S. Pandey, R. Singh, V. Tiwari, K. Sad and V. Tandon, Sci. Rep., 2017, 7, 44419.

19 C. Chen and K. Yang, Bioorg. Chem., 2019, 93, 103286.

20 S. Sabatini, M. Piccioni, T. Felicetti, S. De Marco, G. Manfroni, R. Pagiotti, M. Nocchetti, V. Cecchetti and D. Pietrella, RSC Adv., 2017, 7, 37007-37014.

21 J. W. Park, S. Y. Lee, J. Y. Yang, H. W. Rho, B. H. Park, S. N. Lim, J. S. Kim and H. R. Kim, Biochim. Biophys. Acta, 1997, 1344, 132-138.

22 A. Mahamoud, J. Chevalier, S. Alibert-Franco, W. V. Kern and J. M. Pagès, J. Antimicrob. Chemother., 2007, 59, 1223-1229.
23 K. Kansaku, S. Takeo, N. Itami, A. Kin, K. Shirasuna, T. Kuwayama and H. Iwata, PLoS One, 2017, 12(11), e0188099.

24 X. Zhang, Y. Zhang, F. Wang, C. Wang, L. Chen, H. Liu, H. Lu, H. Wen and T. Zhou, Int. J. Antimicrob. Agents, 2018, 52(2), 226-232.

25 J. Fedorowicz, J. Saczewski, A. Konopacka, K. Waleron, D. Lejnowski, K. Ciura, T. Tomašič, Ž. Skok, K. Savijoki, M. Morawska, S. Gilbert-Girard and A. Fallarero, Eur. J. Med. Chem., 2019, 179, 576-590.

26 I. Wiegand, K. Hilpert and R. E. Hancock, Nat. Protoc., 2008, 3(2), 163-175.

27 K. Xu, S. He, S. Chen, G. Qiu, J. Shi, X. Liu, X. Wu, J. Zhang and W. Tang, Eur. J. Med. Chem., 2018, 154, 144-154. 